\title{
Session 10: Applied technologies I
}

Wednesday 31st October 2007. Moderator: To be confirmed

[10.50-11.35]

[Keynote lecture]

Antibody targeting of stem cells

Jim W. Larrick

Panorama Research, Mountain View, California, USA

Abstracts not provided.

\section{[11.35-11.55]}

\section{Development of recombinant polyclonal antibodies John Haurum \\ Symphogen A/S, Elektrovej, Building 375, DK-2800 \\ Lyngby, Denmark}

Symphogen technologies for development of targetspecific fully human recombinant polyclonal antibody therapeutics are based on site-specific integration to generate robust polyclonal antibody producer cell lines. Development challenges with respect to chemistry, manufacturing and control of recombinant polyclonal antibodies will be reviewed and discussed. The presentation will be based on the development of Sym001, a recombinant polyclonal anti-rhesus $\mathrm{D}$ antibody which is in clinical development for therapy of ITP and prophylaxis of hemolytic disease of the newborn.

\section{[11.55-12.15]}

UniBody: A novel human antibody-based platform for immunotherapy

Janine Schuurman, Tom Vink, Aran Labrijn, Wim Bleeker, Patrick van Berkel, Jan G.J. van de Winkel and Paul W.H.I. Parren

Genmab, Utrecht, The Netherlands

Immunotherapy with monoclonal antibodies is revolutionizing the treatment of cancer and inflammatory diseases. However, it is becoming clear that classical human antibodies do not always represent the best therapeutic modality. Certain therapeutic targets and disease indications may instead be more optimally addressed with inert antibody formats. Genmab's UniBody is representing a breakthrough in this development. It provides the first example of a univalent (noncross-linking) human antibody format which does not activate $\mathrm{Fc}$-mediated effector function, but which retains the long in vivo half life of classical IgG antibodies.

\section{[12.15-12.35]}

Treatment of experimental autoimmune myasthenia gravis with human $\operatorname{IgG}_{4}$ anti acetylcholine receptor competitor antibodies M.H. De Baets, M .Losen, P. Martínez-Martínez and P. Parren ${ }^{4}$

Research Institute Brain and Behaviour, University of Maastricht, The Netherlands

${ }^{4}$ The Netherlands.Genmab, Utrecht, The Netherlands

Autoantibodies against the main immunogenic region (MIR) of the acetylcholine receptor (AChR) play a prominent role in the initiation and pathology of myasthenia gravis and have been suggested to induce episodes of muscle weakness by complement-mediated destruction of the postsynaptic membrane and downregulation of the AChR. In a rhesus monkey model, we show that MG can be induced by passive transfer of a recombinant anti-MIR antibody of the human IgG1 subclass derived from a MG patient. The induction of the disease was prevented by simultaneous injection of an anti-MIR antibody of the IgG4 subclass. No changes in neuromuscular transmission as observed by electromyography, or endplate structure and integrity as observed by electronmicroscopy were detected. The mechanism of protection was analyzed in vitro using the rhabdomyosarcoma cell-line TE671. The results suggest that internalization of the AChR caused by bivalent binding of human IgG1 antibodies are an essential mechanism for induction of MG while the monovalent IgG4 antibodies are protective. The results are sup- 
ported by the observation that an increase of the AChR anchoring protein rapsyn prevents $\mathrm{MG}$ by a reduction of AChR internalization.

\section{[12.35-12.55]}

\section{The sclerostin antibody project Martyn Robinson \\ UCB-Celltech, Slough, UK}

DNA sequencing has allowed the identification of approximately 25,000 genes in the human genome. This number is smaller than predicted but still a frighteningly large number for those individuals charged with identifying the best new targets for the next generation of therapeutics. The degree of validation that each new target requires is also increasing. It was once enough to identify a gene encoding a product that was causally involved in a disease process to trigger a major research effort. However, new drugs such as the anti-TNFs have raised treatment standards in a number of diseases and increasingly, questions are being asked even at the early research stage about the potential of new points of therapeutic intervention to improve on existing therapies (or those currently in development). The challenges of answering this sort of question and identifying new therapeutic targets with commercial potential have probably never been greater.

There are a number of ways that research efforts can be focussed on targets that have a higher probability of being useful points for therapeutic intervention. One of these

is the so called "gene to drug" approach to target identification. This approach was widely discussed in the late 1990s but has not yielded many successful drug development projects to date. In concept, the idea was to use knowledge of the effects of gene mutations to allow the identification of new and useful points of therapeutic intervention. Whilst the approach does not seem to have been generally successful it has a number of attractions, not least that it can allow research efforts to be focussed on gene products whose perturbation produce a known phenotype.

The sclerostin antibody project arose from the "gene to drug" approach and evolved from the study of a rare inherited disease found predominantly in an Afrikaner population in South Africa [1]. The affected individuals have very high bone density and notably strong bones (there are no recorded reports of a traumatic fracture in individuals suffering from sclerosteosis) whilst suffering from few clinical issues unconnected with excessive bone deposition. Studies on the sclerosteosis patients suggested that the high bone mineral density was due to increased bone formation rates rather than a major suppression of bone resorption [2]. It was recognised that this phenotype was one that it would be desirable to replicate in patients suffering from low bone mineral density disorders such as osteoporosis, where bone loss puts patients at increased risk of bone fracture. Fractures caused by osteoporosis are associated with a high mortality and tremendous social costs (the direct health care costs of osteoporotic fractures was estimated at $\$ 18$ billion in 2002 [3]. Whilst most current therapeutic approaches focus on reducing bone loss the idea of being able to stimulate the formation of new high quality bone in patients with low bone mineral density is very attractive.

Genetic mapping and extensive genomic sequencing allowed the identification of the mutation in sclerosteosis patients that resulted their high bone mass phenotype [4]. The mutation inactivates an osteocyte - produced protein called sclerostin that plays an important role in negatively regulating the anabolic output from the osteoblast lineage (and hence regulates bone formation). UCB in collaboration with Amgen have shown that dosing of neutralising antibodies to sclerostin can lead to an increase in bone formation and bone strength in several different species.

Sclerostin was identified following the molecular analysis of a rare inherited human high bone mass disorder and it is hoped that it may represent a target for a new generation of anabolic bone therapies.

\section{References}

[1] H. Hamersama, J. Gardner and P. Beighton, The natural history of sclerosteosis, Clin Genet 63 (2003), 192-197.

[2] National Osteporosis Foundation.

[3] Sclerosteosis: Neurogenetic and pathophysiolgic analysis of an American kinship, S. Stein, C. Witkop, S. Hill, M.D. Fallon, L. Viernstein, G. Gucer, P. McKeever, D. Long, J. Altman, N.R. Miller, S.L. Teitlbaum and S. Schlesinger, Neurology 33 (1983), 267-277.

[4] Bone dysplasia sclerosteosis results from the loss of the SOST gene product, a novel cystine knot-containing protein, $\mathrm{M}$. Brunkow, J.C. Gardner, J. Van Ness, B. Paeper, B.R. Kovacevich, S. Proll, J.E. Skonier, L. Zhoa, P.J. Sabo, Y.-H. Fu, R.S. Alisch, L. Gillet, T. Colbert, P. Tacconi, D. Galas, H. Hamersama, P. Beighton and J.T. Mulligan, Am J Genet 68 (2001), 577-589. 